\title{
Ultrathin flexible bronchoscopy in neonatal intensive care units
}

\author{
Jacques de Blic, Christophe Delacourt, Pierre Scheinmann
}

\begin{abstract}
Thirty seven flexible bronchoscopies were performed in 33 infants in a neonatal intensive care unit, using a $2.2 \mathrm{~mm}$ flexible ultrathin bronchoscope. Twenty eight procedures were performed via an endotracheal tube or tracheostomy and nine in spontaneously breathing infants. Indications for endoscopy included persistent atelectasis and/or emphysema $(n=21)$, unexplained acute respiratory distress $(n=10)$, stridor $(n=3)$, assessment of congenital abnormalities of the tracheobronchial tree $(n=2)$, and follow up of an endobronchial granuloma during the course of corticosteroid treatment $(n=1)$. Abnormal airway dynamics and/or abnormal structure were seen in 23 of 37 cases. In $54 \%$ of the procedures, the results of bronchoscopy had a direct effect on further management. The procedure was well tolerated and completed in less than two minutes. Our results suggest that the ultrathin flexible bronchoscope improves airway exploration and the understanding of respiratory disorders during the first months of life, particularly in ventilated infants.
\end{abstract}

Persistent lung atelectasis, localised hyperinflation, and acute respiratory failure are common events in neonatal intensive care units (NICU). They constitute well accepted indications for urgent bronchoscopy. ${ }^{1}$ Direct examination of the airways is the definitive diagnostic procedure in infants with bronchopulmonary dysplasia, ${ }^{2}$ congenital heart and/or lung anomalies, ${ }^{34}$ and generally in intubated mechanically ventilated infants. Because of the small internal diameter of the airways and/or of the endotracheal tube ultrathin flexible bronchoscopes have been developed. ${ }^{5-8}$ Valuable information is thus provided concerning patency and the position of the endotracheal tube ${ }^{589}$ and in evaluating tracheal injury. ${ }^{10}$ This report describes our experience with an Olympus $2 \cdot 2 \mathrm{~mm}$ flexible bronchoscope in the assessment of airway obstruction by malacia, intrinsic obstacles, or extrinsic masses or vessels.

\section{Patients and methods}

Between October 1989 and April 1990, we performed 37 flexible bronchoscopies in 33 infants. The mean age at presentation was 94 days (range 2 days to 9 months) and the mean weight $4 \mathrm{~kg}$ (range 1010-7000 g).

Twenty seven infants had an underlying disease: bronchopulmonary dysplasia $(n=9)$, congenital heart disease $(n=10)$, congenital malformation of the lower respiratory tract $(n=6)$ including scimitar syndrome, pulmonary artery 'sling', bronchopulmonary sequestration, left diaphragmatic hernia with pulmonary hypoplasia, and two infants with tracheo-oesophageal fistula with oesophageal atresia; one child had bullous epidermolysis and one had cystic fibrosis. The remaining six children ranged in age from 6 to 22 days and had been admitted to the NICU for the sole diagnosis of neonatal respiratory distress.

Flexible bronchoscopy was performed with a prototype flexible ultrathin bronchoscope with an outer diameter of $2.2 \mathrm{~mm}$ (model XBF22 Olympus Corporation). This bronchoscope is immersible, it has an angle of flexion of $130^{\circ}$ and an angle of antiflexion of $65^{\circ}$, but no suction channel.

Twenty six of the 37 endoscopies were carried out in intubated children. The diameters of the endotracheal tubes were as follows: $2.5 \mathrm{~mm}$ $(\mathrm{n}=4), 3 \mathrm{~mm}(\mathrm{n}=11), 3.5 \mathrm{~mm}(\mathrm{n}=9)$, and $4 \mathrm{~mm}$ $(n=4)$. The flexible bronchoscope was passed through a swivel Y connector (Vigon, France) located between the endotracheal tube and the ventilator. The adaptor is specially fitted with a cleft to accommodate the bronchoscope and thus allows uninterrupted mechanical ventilation and oxygen delivery. In two cases the bronchoscope was passed through a tracheostomy stoma. No sedation was used before the procedure. All bronchoscopies were performed after preoxygenation in order to obtain an arterial oxygen saturation $\left(\mathrm{SaO}_{2}\right)$ greater than $90 \%$. Except for changes in fractional inspired oxygen, ventilator settings remained unchanged during the procedure.

Nine infants were ventilating spontaneously but their weight $(<2500 \mathrm{~g})$ and/or their respiratory status precluded the use of a conventional paediatric flexible broncoscope (external diameter $3.6 \mathrm{~mm}$ ). Bronchoscopies were performed transnasally with an ultrathin flexible bronchoscope, according to the technique used in older children, ${ }^{11}$ with sedation and topical anaesthesia of upper airways with lignocaine. Special care was taken to apply suction carefully to the upper airways as the instrument has no operator channel. The cross section of the XBF22 is approximately one third that of the paediatric bronchofibrescope $\left(3.8 \mathrm{~mm}^{2} v\right.$ $10 \mathrm{~mm}^{2}$ ), thus allowing sufficient spontaneous ventilation, even in low birthweight neonates. All flexible bronchoscopies were performed in the NICU. Heart rate and oxygen saturation (Ohmeda), were continuously monitored in all patients during the procedures. 
Indications and findings of 37 ultrathin flexible bronchoscopies in a NICU

\begin{tabular}{|c|c|c|}
\hline Indications & $\begin{array}{l}\text { No of } \\
\text { cases }\end{array}$ & Findings \\
\hline Persistent atelectasis/emphysema & 21 & $\begin{array}{l}\text { Normal }(n=2) \text {, granuloma }(n=2) \text {, inflammatory stenosis }(n=3) \text {, } \\
\text { bronchomalacia }(n=5) \text {, vascular compression }(n=5) \text {, } \\
\text { foreign body }(n=1) \text {, hypersecretion }(n=3) \text {, tracheal bronchus } \\
(n=2)^{*}\end{array}$ \\
\hline Acute respiratory distress & 10 & $\begin{array}{l}\text { Normal }(n=5) \text {, granuloma }(n=1) \text {, bronchomalacia }(n=2) \text {, } \\
\text { tracheomalacia }(n=1) \text {, hypersecretion }(n=1) \dagger\end{array}$ \\
\hline Stridor & 3 & $\begin{array}{l}\text { Tracheomalacia }(n=1) \text {, bronchomalacia }(n=1) \text {, laryngeal } \\
\text { bulla }(n=1)\end{array}$ \\
\hline $\begin{array}{l}\text { Congenital abnormalities of tracheobronchial treeł } \\
\text { Control }\end{array}$ & $\begin{array}{l}2 \\
1\end{array}$ & $\begin{array}{l}\text { Normal }(n=1) \text {, vascular compression }(n=1) \\
\text { Normal }(n=1)\end{array}$ \\
\hline
\end{tabular}

*Associated tracheomalacia in one case and inflammatory stenosis of the right intermediate bronchus in the other case.

†Airway examination impossible.
¡Isolated (without atelectasis or emphysema) pulmonary sequestration and pulmonary artery 'sling'.

Indications for endoscopic examination (see table) included the suspicion or assessment of obstacles caused by vascular compression in children with congenital heart-vessel abnormalities or by stenosis, granuloma, or tracheobronchomalacia in long term mechanically ventilated infants. Most procedures were carried out secondary to persistent atelectasis and/or emphysema $(n=21)$ or in instances of unexplained acute respiratory distress $(n=10)$. Other indications for ultrathin bronchoscopy included stridor $(n=3)$, assessment of congenital abnormalities of the tracheobronchial tree $(n=2)$, and follow up of an endobronchial granuloma during the course of corticosteroid treatment $(n=1)$. In seven cases flexible bronchoscopy was performed in the early postoperative period: after cardiac surgery $(n=5)$ and after repair of the left diaphragmatic hernia $(n=1)$ and of an oesophageal atresia $(n=1)$.

\section{Results}

In 9/37 cases flexible bronchoscopy excluded suspected extrinsic or intrinsic obstruction of the airways (see table). The bronchoscopy confirmed laryngeal lesions in a 2 month old infant with bullous epidermolysis.

On four occasions massive bronchial hypersecretion was documented. In one case, vision was so blurred that airway examination was impossible. In another case, a mucous plug located in the intermediate bronchus had to be subsequently removed with a rigid bronchoscope.

In 23 of 37 cases, abnormal airway dynamics and/or abnormal structure were seen. Severe tracheomalacia and/or bronchomalacia were observed on 10 occasions (bronchopulmonary dysplasia in three, congenital heart anomalies in four, scimitar syndrome in two, and after tracheo-oesophageal fistula remain in one). Airway compression by enlarged heart cavities or vessels was found in five cases of congenital heart anomalies and in the case of pulmonary artery 'sling'. Using ultrathin bronchoscopy we documented three cases of inflammatory stenosis of the right intermediate bronchus, three occluding granulomas (one tracheal and two bronchial) as well as one case of a foreign body in the right intermediate bronchus. Two infants with right upper lobe atelectasis had a tracheal bronchus: one of them was examined soon after repair of an oesophageal atresia and had associated tracheomalacia; the second, a $1100 \mathrm{~g}$ premature infant, had a right lower lobe emphysema, and inflammatory stenosis of the right $\vec{O}$ intermediate bronchus. The diagnostic yield of ultrathin flexible bronchoscopy was higher in $\omega_{\sigma}$ cases of radiologically documented atelectasis or emphysema $(80 \%)$ than in cases where these ? findings were absent $(5 / 10 ; 50 \%)$.

The procedure was well tolerated and completed in less than two minutes. The decrease of $\mathrm{SaO}_{2}$ was moderate $\left(\mathrm{SaO}_{2}\right.$ remained $\left.>80 \%\right)$, and resolved rapidly after removal of the fibrescope. No bradycardia was observed. None of the spontaneously breathing infants showed $\vec{\nabla}$ desaturation or bradycardia during the procedure.

In $54 \%$ of the procedures, the result of flexible bronchoscopy had a direct effect on further management: earlier correction of $\vec{\theta}$ cardiovascular abnormalities $(n=5)$, extraction of the foreign body $(n=1)$, removal of a mucous $\square$ plug by rigid bronchoscopy $(n=1)$, extreme care in suctioning (no suction below the end of the endotracheal tube), a course of steroids treat-

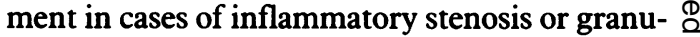
lation tissue $(n=6)$, change in ventilator settings $\overrightarrow{\vec{B}}$ in mechanically ventilated infants and in chest $\exists$ physiotherapy in spontaneously ventilated infants with severe tracheobronchomalacia $(\mathbf{n}=7)$.

\section{Discussion}

Our results confirm the safety and the diagnostic value of ultrathin flexible bronchoscopy in the assessment of airways in newborn and premature infants.

Evidence of persistent, clinical or radiological, airway obstruction is a constant concern $N$ in the NICU and the need for rapid evaluation $N$ of the airways constitutes a major indication for N flexible bronchoscopy. However, until recently the procedure required extubation because of either the size of the paediatric bronchoscope ${ }^{12}$ or the need for rigid bronchoscopy, ${ }^{13}$ thereby greatly limiting the use of such exploration. $T$ With the new ultrathin flexible bronchoscope, extubation is no longer necessary for airway exploration. Previous studies demonstrated the usefulness of this procedure, but the study groups consisted of older ${ }^{14}$ and/or nonintubated children, ${ }^{8}$ without concomitant pathology. Other studies involved the use of either bigger bronchoscopes ${ }^{5} 8$ or an instrument with a non-directed tip, 56914 and the procedures were usually performed in order to 
verify the position of the endotracheal tube. In contrast, $80 \%$ of our patients had concomitant pathology favouring the occurrence of airway obstruction.

The addition of a connector allows the introduction of the $2.2 \mathrm{~mm}$ bronchoscope into the endotracheal tube, maintaining good oxygenation and ventilation, in order to achieve a more comfortable and complete examination. Our results correspond with those of Schellhase et al, who used the same technique and showed that after preoxygenation the procedure was well tolerated, with no serious adverse cardiovascular effects. ${ }^{10}$ Transient hypoxaemia $\left(\mathrm{SaO}_{2}\right.$ $>82 \%$ ) and bradycardia occurred during bronchoscopy and resolved spontaneously in less than 1 min. Ultrathin fibroscopy is well tolerated provided certain precautions are taken: it must be done in an intensive care unit, by an experienced paediatric bronchoscopist, rapidly and under cardiac and oxymetric monitoring. The absence of an operator channel prevents the suctioning of secretions, and the administration of anaesthesia or normal saline. However, careful suctioning before bronchoscopy adequately prepares the airways for the procedure. Bronchial hypersecretion precluded airway examination in only one case in our series.

Our results proved the utility of the ultrathin flexible bronchoscopy in both mechanically ventilated and spontaneously ventilating infants. When searching for airway obstruction in the presence of persistent radiological abnormalities in mechanically ventilated infants (particularly in cases of bronchopulmonary dysplasia and cardiothoracic malformations), we found that in $80 \%$ of the cases the ultrathin bronchoscope showed either endoluminal abnormalities (granuloma, inflammatory stenosis), malformation (tracheal bronchus), severe extrinsic compression, or severe tracheomalacia and/or bronchomalacia. These airway anomalies can exist concurrently and their correct diagnosis is of paramount importance in influencing management decisions and providing information for possible surgical intervention. The change in the management in $54 \%$ of our cases is a strong argument for its use. The findings of significant airway compression by a large vessel may hasten a surgical decision in infants with congenital cardiopathies. In the presence of inflammatory lésions, granuloma or tracheobronchial stenosis, due to prolonged ventilation and repeated suctioning, the suction catheter should not be passed beyond the end of the endotracheal tube, and a course of oral steroids may be given. Lastly, diagnosis of severe tracheobronchomalacia in a mechanically ventilated infant may require positive end expiratory pressure. ${ }^{2}$ Persistent radiological abnormalities are a clear but not the sole indication for ultrathin flexible bronchoscopy. The sudden unexplained deterioration of respiratory status provides a setting in which the fibrescope offers valuable information for proper patient management. ${ }^{6}$ Malacia and granuloma without radiological changes were particularly common in our series.

Classical paediatric flexible bronchoscopy in spontaneously ventilating infants carries the risk of inducing respiratory failure especially in small patients $(\leqslant 2500 \mathrm{~g})$ or in those with borderline respiratory status. Ultrathin bronchoscopy offers a safe alternative in the above mentioned circumstances.

In conclusion, the $2 \cdot 2 \mathrm{~mm}$ ultrathin flexible bronchoscope is very useful in a NICU in improving airway exploration and the understanding of respiratory disorders during the first months of life, particularly in ventilated infants.

1 Wood RE, Postma D. Endoscopy of the airway in infants and children. F Pediatr 1988;112:1-6.

2 Cohn RC, Kercsmar C, Dearborn D. Safety and efficacy of flexible bronschoscopy in children with bronchopulmonary dysplasia. Am 7 Dis Child 1988;142:1225-8.

3 Benjamin B. Endoscopy in congenital tracheal anomalies. f Pediatr Surg 1980;15:164-71.

4 Kosloske AM, Jewell PF, Cartwright KC. Crucial bronchoscopic findings in esophageal atresia and tracheoesophagea fistula. F Pediatric Surg 1988;23:466-70.

5 Finer NN, Etches PC. Fiberoptic bronchoscopy in the neonate. Pediatr Pulmonol 1989;7:116-20.

6 Myer MM, Thompson RF. Flexible fiberoptic bronchoscopy in the neonatal intensive care unit. Int $\mathcal{f}$ Pediatr Otorhinolaryngol 1988;15:143-7.

7 Wood RE. Clinical applications of ultrathin flexible bronchoscopes. Pediatr Pulmonol 1985;1:244-8.

8 Fan LL, Sparks LM, Dulinski JP. Applications of an ultrathin flexible bronchoscope for neonatal and pediatric airway problems. Chest 1986;89:673-6.

9 Shinwell ES, Higgins RD, Auten RL, Shapiro DL. Fiberoptic bronchoscopy in the treatment of intubated neonates. optic bronchoscopy in the treatment
Am $\mathcal{F}$ Dis Child 1989;143:1064-5.

10 Schellhase DE, Graham LM, Fix EJ, Sparks LM, Fan LL. Diagnosis of tracheal injury in mechanically ventilated Diagnosis of tracheal injury in mechanically ventilated
premature infants by flexible bronchoscopy. A pilot study. premature infants by flexil

11 de Blic J, McKelvie P, Le Bourgeois M, Blanche S, Benois MR, Scheinmann $P$. Value of bronchoalveolar lavage in the management of severe acute pneumonia and interstitia pneumonitis in the immunocompromised child. Thorax 1987;42:759-65.

12 de Blic J, Scheinmann P, Paupe J. Successful treatment of persistent neonatal emphysema by flexible bronchoscope. Lancet 1984;ii: 1389-90.

13 Muntz HR. Therapeutic rigid bronchoscopy in the neonata intensive care unit. Ann Otol Rhinol Laryngol 1985;94: 462-5.

14 Ward RF, Arnold FE, Healy GB. Flexible minibronchoscopy in children. Ann Otol Rhinol Laryngol 1987;96:645-9. 